\title{
Ultrafast magnetization enhancement in metallic multilayers driven by superdiffusive spin current
}

\author{
Dennis Rudolf1, ${ }^{1,}$, Chan La-O-Vorakiat ${ }^{2, \star}$, Marco Battiato ${ }^{3, \star}$, Roman Adam ${ }^{1}$, Justin M. Shaw ${ }^{4}$, Emrah Turgut ${ }^{2}$, \\ Pablo Maldonado ${ }^{3}$, Stefan Mathias ${ }^{2,5}$, Patrik Grychtol ${ }^{1,2}$, Hans T. Nembach ${ }^{4}$, Thomas J. Silva4, \\ Martin Aeschlimann ${ }^{5}$, Henry C. Kapteyn², Margaret M. Murnane ${ }^{2}$, Claus M. Schneider ${ }^{1}$ \& Peter M. Oppeneer ${ }^{3}$
}

Uncovering the physical mechanisms that govern ultrafast charge and spin dynamics is crucial for understanding correlated matter as well as the fundamental limits of ultrafast spin-based electronics. Spin dynamics in magnetic materials can be driven by ultrashort light pulses, resulting in a transient drop in magnetization within a few hundred femtoseconds. However, a full understanding of femtosecond spin dynamics remains elusive. Here we spatially separate the spin dynamics using $\mathrm{Ni} / \mathrm{Ru} / \mathrm{Fe}$ magnetic trilayers, where the $\mathrm{Ni}$ and Fe layers can be ferroor antiferromagnetically coupled. By exciting the layers with a laser pulse and probing the magnetization response simultaneously but separately in $\mathrm{Ni}$ and $\mathrm{Fe}$, we surprisingly find that optically induced demagnetization of the Ni layer transiently enhances the magnetization of the Fe layer when the two layer magnetizations are initially aligned parallel. Our observations are explained by a laser-generated superdiffusive spin current between the layers.

\footnotetext{
${ }^{1}$ Peter Grünberg Institut PGI-6 \& JARA-FIT, Research Centre Jülich, 52425 Jülich, Germany. ${ }^{2}$ Department of Physics and JILA, University of Colorado and NIST, Boulder, Colorado, USA. ${ }^{3}$ Department of Physics and Astronomy, Uppsala University, SE-75120 Uppsala, Sweden. ${ }^{4}$ Electromagnetics Division, National Institute of Standards and Technology, Boulder, Colorado, USA. ${ }^{5}$ University of Kaiserslautern and Research Center OPTIMAS, 67663 Kaiserslautern, Germany. ${ }^{\star}$ These authors contributed equally to this work. Correspondence and requests for materials should be addressed to R.A. (email: r.adam@fz-juelich.de).
} 
O ne of the major roadblocks for continued progress in nanoelectronics is energy dissipation caused by the flow of electrical current. Encoding data in electron spin, rather than charge, promises a new route for technology with the potential to dramatically reduce these energy requirements. A current grand challenge in this area is to control the static and dynamic behaviour of spins and spin ensembles. Spin dynamics is in-itself of fundamental interest for understanding spin-photon-charge-phonon interactions and their complex interplay on femtosecond to picosecond timescales. The potential to rapidly manipulate spins was first demonstrated in a pioneering experiment ${ }^{1}$ on $\mathrm{Ni}$ that observed that the magnetization of $3 d$ ferromagnets can be optically quenched within $50-300 \mathrm{fs}$ after excitation by a femtosecond laser pulse $\mathrm{e}^{2,3}$. This ultrafast demagnetization is then followed by a slower magnetization recovery on picosecond timescales. Optically induced magnetization reversal has also been demonstrated using circularly polarized light ${ }^{4}$. A number of distinct models have been proposed to describe how laser excitation can couple so quickly to the spins, given that the light itself does not directly exert a significant torque on the electron spins. Most of these models are based on phonon-, electronand magnon-mediated spin-flip processes ${ }^{5-9}$, direct laser-induced spin-flips ${ }^{10}$ or relativistic spin-light interaction ${ }^{11}$. Most recently, a new model based on superdiffusive spin transport has been proposed $^{12,13}$.

The variety of these distinct interpretations suggests that several physical mechanisms may govern femtosecond spin dynamics even in a simple elemental magnet. The physics becomes even more intriguing for heterogeneous magnetic systems, such as magnetic compounds $^{14}$, alloys ${ }^{15,16}$ and exchange-coupled layered structures. Several sophisticated experiments have recently employed ultrashort soft X-ray pulses from synchrotron ${ }^{2,15}$ or laser-driven high-harmonic (HHG) light sources ${ }^{3,16,17}$ to disentangle the various processes using element-specific demagnetization techniques. This has been achieved by monitoring the absorption or reflection of light tuned to inner-shell absorption edges of magnetic materials. Using these new capabilities, unexpected new spin dynamics can be uncovered-for example, even in a strongly coupled ferromagnetic permalloy $\left(\mathrm{Fe}_{20} \mathrm{Ni}_{80}\right)$, the magnetization of $\mathrm{Fe}$ quenches faster than the Ni magnetization for a time shorter than the characteristic energy/time equivalent of the exchange coupling energy of the alloy $^{16}$. In magnetic multilayers, magnetization dynamics has been previously studied on nanosecond to picosecond timescales ${ }^{18-20}$. However, accurately explaining how multilayer systems demagnetize on femtosecond timescales has proven challenging. Recent exciting experiments optically pumped layered magnetic structures using femtosecond lasers 21,22 and inferred the resulting spin transport dynamics using visible probe light.

Here we exploit the combination of simple magnetic multilayer structures and the powerful capability of our HHG probe to simultaneously monitor layer-specific magnetization dynamics. We study a Ni/Ru/Fe trilayer stack under conditions where superdiffusive spin transport is the dominant process determining spin dynamics on the fastest timescales. By optically exciting the stack with femtosecond laser pulses and probing the resulting magnetization dynamics in the $\mathrm{Ni}$ and Fe layers simultaneously but element-selectively at the $3 p(\mathrm{M})$ absorption edges of $\mathrm{Ni}(66 \mathrm{eV})$ and $\mathrm{Fe}(52 \mathrm{eV})$, we directly observe magnetization dynamics in individual layers. Remarkably, we find that the femtosecond spin dynamics of the individual $\mathrm{Ni}$ and Fe layers depends on the relative alignment of their initial magnetization directions: ultrafast excitation and demagnetization of the $\mathrm{Ni}$ layer transiently enhances the magnetization of the buried $\mathrm{Fe}$ layer when their magnetizations are initially aligned parallel to each other, whereas the magnetization of the Fe layer decreases if its initial magnetization is antiparallel to that of $\mathrm{Ni}$. These findings can be explained consistently by large laser-generated superdiffusive spin currents $^{12,13}$. In this physical picture, superdiffusion of excited spin

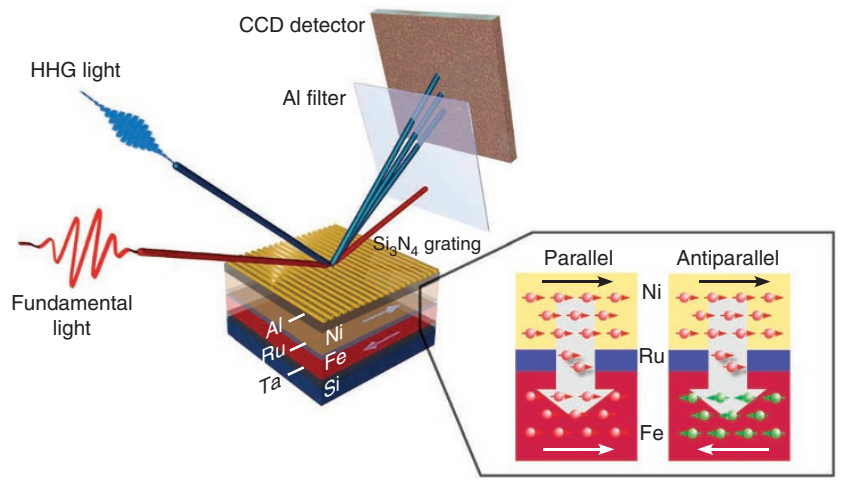

Figure 1 | Experimental setup and the multilayer sample schematics. The magnetization of the $\mathrm{Al} / \mathrm{Ni} / \mathrm{Ru} / \mathrm{Fe}$ multilayer samples is probed by XUV high harmonics at the $3 p$ absorption edges of $\mathrm{Fe}$ and $\mathrm{Ni}$. The $\mathrm{Si}_{3} \mathrm{~N}_{4}$ grating on top of the layers spectrally separates the XUV high harmonic beams that are then detected by an X-ray CCD detector. The pump laser light absorbed in the thin $\mathrm{Al}$ and Ni layers results in a spin current (marked by grey arrow in the inset) that travels through the non-magnetic Ru spacer layer into the Fe layer. The magnetization of the Fe layer increases or decreases depending on its orientation relative to $\mathrm{Ni}$. The inset illustrates schematically the relative magnetization of the $\mathrm{Ni}$ and Fe layers (thin black and white arrows), the majority spin alignment in the layers (red and green circles) and the flow of the spin current (large grey arrow).

majority electrons from the Ni layer, through the Ru layer and into the buried Fe layer can transiently increase or decrease the magnetization in Fe depending on its relative spin alignment with respect to $\mathrm{Ni}$ (which can be controlled by the external magnetic field and the thickness of the Ru spacer layer).

\section{Results}

Multilayer fabrication and characterization. Our samples consist of $\mathrm{Al}(3 \mathrm{~nm}) / \mathrm{Ni}(5 \mathrm{~nm}) / \mathrm{Ru}(x) / \mathrm{Fe}(4 \mathrm{~nm}) / \mathrm{Ta}(3 \mathrm{~nm})$ layers (see Methods), where the $\mathrm{Ni} / \mathrm{Ru} / \mathrm{Fe}$ trilayer forms an interlayer exchangecoupled magnetic system (Fig. 1). The top $\mathrm{Si}_{3} \mathrm{~N}_{4}$ grating structure serves as a grating spectrometer to spectrally disperse the extreme ultraviolet (XUV) HHG radiation spanning from approximately 20 to $72 \mathrm{eV}$. The magnetism in our samples was carefully characterized using a superconducting quantum interference device (SQUID) magnetometer. These data (see Fig. 2) show the influence of the $\mathrm{Ru}$ spacer thickness on the interlayer exchange-coupling between $\mathrm{Ni}$ and Fe layers: a $1 \mathrm{~nm}$ thick $\mathrm{Ru}$ layer results in a groundstate ferromagnetic coupling for $\mathrm{Ni}$ and $\mathrm{Fe}$, which changes to antiferromagnetic coupling for a $1.5 \mathrm{~nm}$ Ru layer thickness.

Static magnetic asymmetry. The magneto-optical response of our trilayers is measured via the transverse magneto-optical Kerr effect (T-MOKE) in XUV spectral range ${ }^{23-25}$ (see Supplementary Methods for a more detailed description). Magnetic asymmetry can be defined as

$$
A=\frac{I_{r}\left(H^{\uparrow}\right)-I_{r}\left(H^{\downarrow}\right)}{I_{r}\left(H^{\uparrow}\right)+I_{r}\left(H^{\downarrow}\right)}
$$

where $I_{r}\left(H^{\uparrow}\right)$ and $I_{r}\left(H^{\downarrow}\right)$ denote the intensities of the reflected light as the externally applied magnetic field $H$ is reversed in direction. Measuring $I_{r}(H)$ for these two opposite field directions gives an artifact-free and sensitive measurement of the magnetic state ${ }^{16,17,26}$. The resonantly enhanced static magnetic asymmetry at the Fe and Ni $3 p$ absorption edges ( 52 and $66 \mathrm{eV}$, respectively) can be clearly assigned to the magnetic state of the Fe and Ni layers, as shown in Fig. 3. For our $\mathrm{Ni} / \mathrm{Ru} / \mathrm{Fe}$ trilayer, the amplitude of the magnetic asymmetry is 

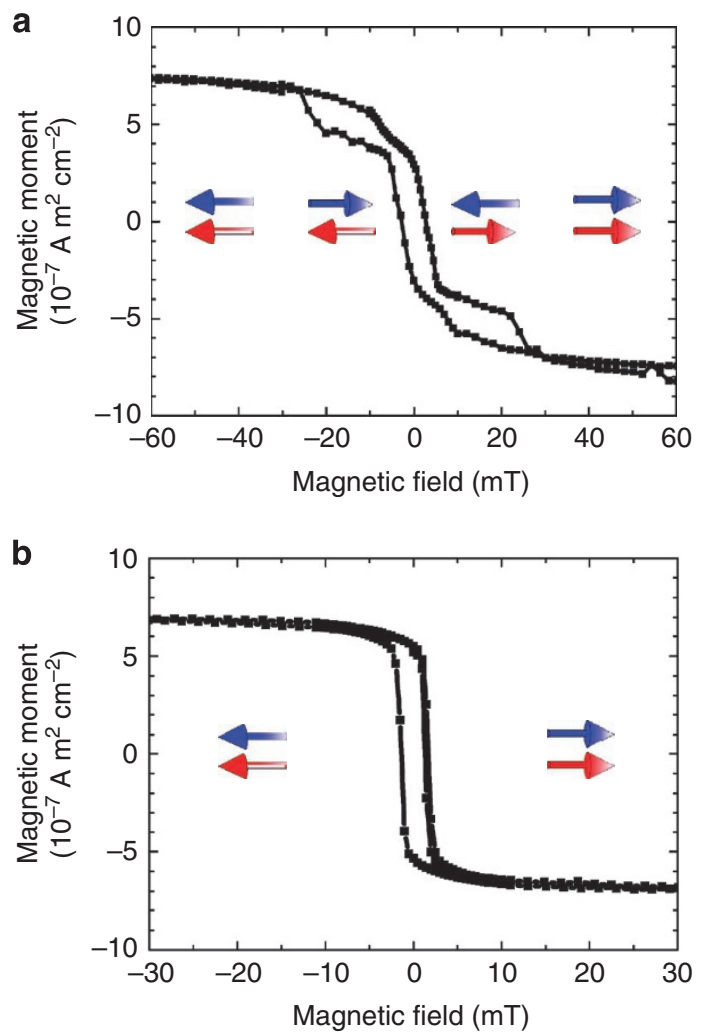

Figure 2 | Magnetic hysteresis loops. The magnetic moment of the $\mathrm{Ni}(5 \mathrm{~nm}) / \mathrm{Ru}(x) / \mathrm{Fe}(4 \mathrm{~nm})$ trilayers, where $x=1 \mathrm{~nm}$ and $1.5 \mathrm{~nm}$, was measured by a SQUID magnetometer at $290 \mathrm{~K}$. The Fe and Ni layers in the trilayer with a Ru thickness of $1.5 \mathrm{~nm}$ (a) are antiferromagnetically coupled in zero external magnetic field, whereas they are ferromagnetically coupled in the $1 \mathrm{~nm}$ thick Ru trilayer (b). The red and blue arrows show the magnetizations of $\mathrm{Fe}$ (red) and $\mathrm{Ni}$ (blue) layers indicating the relative magnetization orientation within a specific magnetic field range.

around $10 \%$ at the $\mathrm{Fe}$ absorption edge and $20 \%$ at the Ni $3 p$ edge. By adjusting the external magnetic field from 0 to $\pm 60 \mathrm{mT}$, we can tune the trilayers into two well-defined magnetic states: one with parallel orientation of the $\mathrm{Ni}$ and $\mathrm{Fe}$ magnetizations and one with antiparallel orientation. A comparison of the magnetic asymmetries corresponding to the two states shows that the relative sign of the $\mathrm{Ni}$ and $\mathrm{Fe}$ asymmetries reverses in these two cases.

Magnetization dynamics. To capture spin transport and magnetization dynamics in our trilayer, the samples are excited with femtosecond pump laser pulses of $1.6 \mathrm{eV}$ photon energy. The magnetization in the individual layers was then captured simultaneously by monitoring the amplitude of the T-MOKE magnetic asymmetry as a function of time delay. To estimate the amount of the pump light reaching the individual layers, we performed a self-consistent $2 \times 2$ matrix calculation of the optical properties for the full metallic layer stack for our experimental conditions. We find that the combination of the $\mathrm{Al}$ cap and Ni layer together absorbs $>58 \%$ of the incident light-about 2.5 times as much as the Fe layer. Generally, as in all optical pump-probe experiments, it is possible that the magnetooptical signal is affected by the hot electrons, in which case the signal would also change sign with switching the magnetization. However, the fact that changing the relative magnetization direction of the Fe layer relative to the Ni layer (see below) causes an increase or decrease of the observed Fe magnetization, rules out such measurement artifacts. In addition, in a previous publication ${ }^{17}$ we showed that the nonmagnetic contribution to the asymmetry parameter
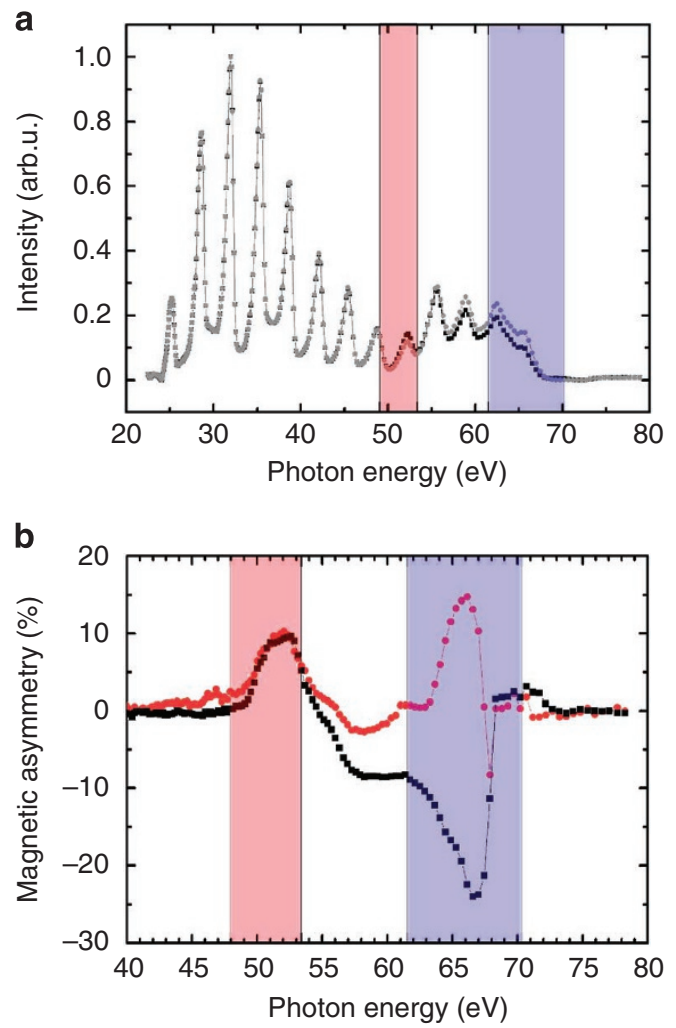

Figure 3 | High harmonics and magnetic asymmetry spectra. (a) Spectra of high harmonics reflected from the sample depend on the orientation of the external magnetic field $\left(\mathrm{H}^{\uparrow}\right.$ or $\left.\mathrm{H}^{\downarrow}\right)$. The spectra are taken for parallel alignment of the $\mathrm{Ni}$ and Fe magnetizations for $\mathrm{H}^{\uparrow}$ (black squares) and $\mathrm{H}^{\downarrow}$ (grey circles). The spectra differ at the $3 p$ absorption edges of $\mathrm{Ni}$ (blue-coloured area) and Fe (red-coloured area). (b) Fe and Ni magnetic contributions can be separated in the magnetic asymmetry calculated from high harmonics spectra (equation (1)). The $3 p$ absorption edges of $\mathrm{Fe}$ and $\mathrm{Ni}$ are marked by red- and blue-coloured areas. For parallel alignment of the $\mathrm{Ni}$ and $\mathrm{Fe}$ magnetizations the $\mathrm{Ni}$ and $\mathrm{Fe}$ asymmetry peaks have different signs (black squares) whereas for the antiparallel alignment (red circles) the sign of the $\mathrm{Ni}$ asymmetry peak reverses.

is small $(0.2 \%)$ compared with the amplitude of demagnetization (20\%), demonstrating that XUV T-MOKE can sensitively probe element-selective spin dynamics.

The layer-selective time traces of the magnetic asymmetries at the $3 p$ absorption edges of $\mathrm{Fe}$ and $\mathrm{Ni}$, at fluencies of $\mathrm{F} \approx 2 \mathrm{~mJ} \mathrm{~cm}^{-2}$, are plotted in Fig. 4a,b. Similar to earlier experiments ${ }^{1,2,3,5}$, we observe a magnetization quenching in the Ni layer of the stack. Surprisingly, however, we also observe either an enhancement or reduction of the Fe magnetization depending on the relative magnetization orientation of the Ni and Fe layers (see Fig. 4a,b): if the Fe and Ni layers are initially oriented antiparallel, then the magnetization of both $\mathrm{Ni}$ and Fe decreases. On the other hand, if the two layers are oriented parallel by an external magnetic field, we observe that the Fe magnetization increases $15 \%$ above its equilibrium value on a similar timescale. In a second layer stack with $\mathrm{Ni}(5 \mathrm{~nm}) / \mathrm{Ru}(1 \mathrm{~nm}) / \mathrm{Fe}(4 \mathrm{~nm})$, where the $\mathrm{Ni}$ magnetization only aligns parallel to the $\mathrm{Fe}$ one (Fig. 4c), we observe again an enhancement in the Fe magnetization after laser excitation.

Superdiffusive spin transport. This first observation of an ultrafast magnetization enhancement in the Fe layer can be explained by superdiffusive spin transport ${ }^{12,13}$ (see also Supplementary Discussion), taking place on timescales comparable to the demagnetization 

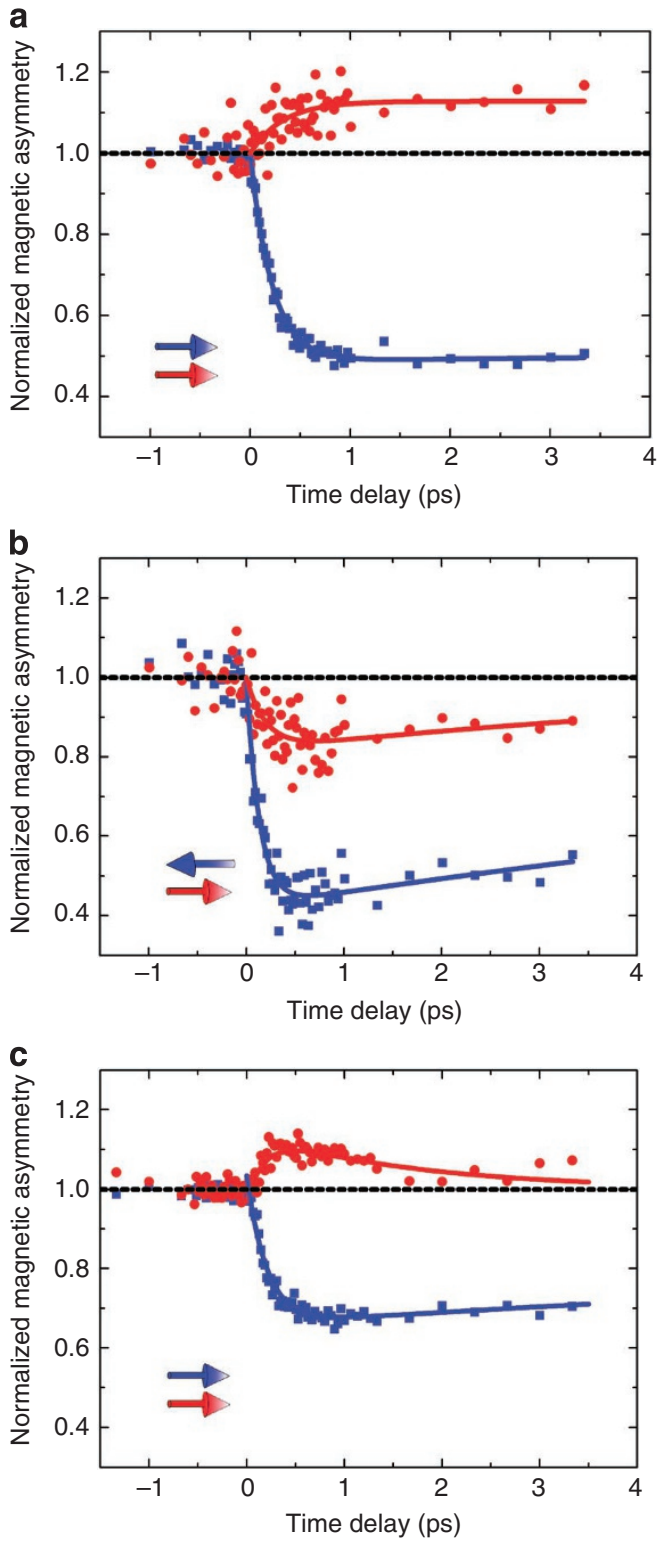

Figure 4 | Experimentally measured time- and layer-resolved magnetization. The time-resolved magnetization of the $\mathrm{Fe}$ and Ni layers in the $\mathrm{Ni}(5 \mathrm{~nm}) / \mathrm{Ru}(1.5 \mathrm{~nm}) / \mathrm{Fe}(4 \mathrm{~nm})$ trilayer for the parallel (a) and antiparallel (b) magnetization alignment and in the $\mathrm{Ni}(5 \mathrm{~nm}) / \mathrm{Ru}(1 \mathrm{~nm}) /$ $\mathrm{Fe}(4 \mathrm{~nm})$ trilayer (c) for the parallel magnetization alignment. The data have been extracted by integrating the magnetic asymmetry signal over the $3 p$ absorption edges of $\mathrm{Fe}$ and $\mathrm{Ni}$, as indicated by the red- and bluecoloured areas in Fig. 3, respectively, and normalizing to the magnetic asymmetry values before the time zero (black broken line). The magnetic asymmetry at the $\mathrm{Fe} 3 p$ absorption edge anomalously increases for the parallel $(\mathbf{a}, \mathbf{c})$ and decreases for antiparallel magnetic orientation of $\mathrm{Ni}$ and Fe layers $(\mathbf{b})$. For the $\mathrm{Ni}(5 \mathrm{~nm}) / \mathrm{Ru}(1 \mathrm{~nm}) / \mathrm{Fe}(4 \mathrm{~nm})$ trilayer, only parallel magnetization orientation is possible due to ferromagnetic coupling. The curves are least square fits to Supplementary Equation (S2).

processes explored in earlier works ${ }^{1-3,5}$. The mechanism we propose for enhancement of the magnetization is based on filling of majority spin states above the Fermi energy in the Fe layer by majority spins coming from $\mathrm{Ni}$. This leads to a transient magnetization increase in the Fe layer, above its maximum value defined by the Curie curve at $T=0 \mathrm{~K}$. The increase in the magnetic signal from the Fe layer is a result of a strong asymmetry in the spin-dependent hot-electron
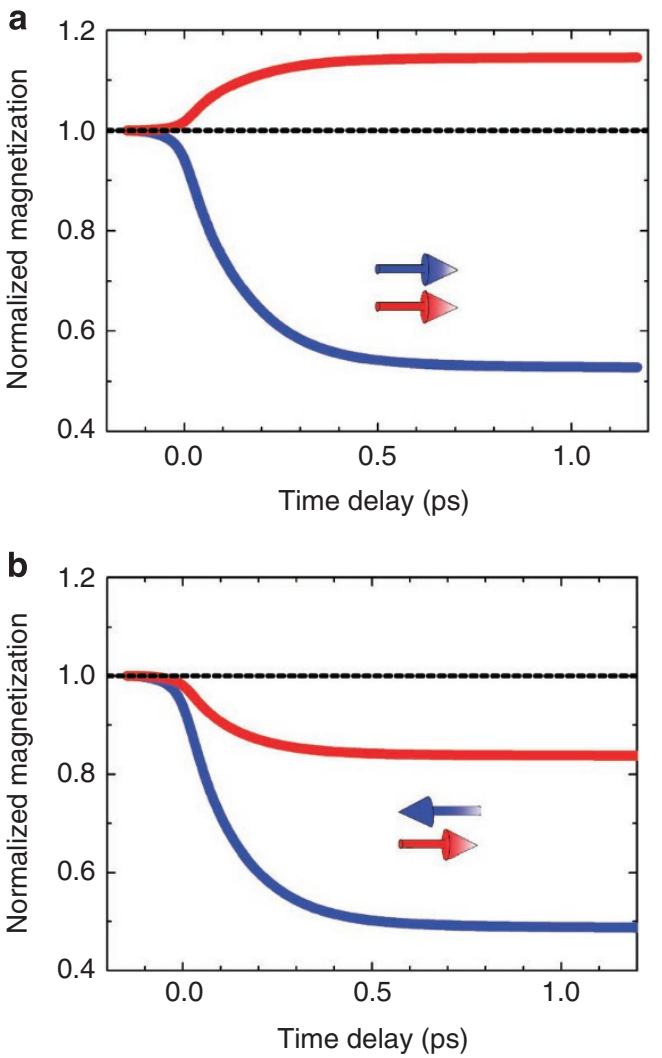

Figure 5 | Calculated time- and layer-resolved magnetization. In our model, laser-induced ultrafast demagnetization of the Ni layer (blue curves in (a) and (b)) generates superdiffusive spin currents that increase the Fe magnetization for parallel orientation (a) (red curve), whereas decreasing the Fe magnetization for antiparallel alignment (b) (red curve). The strengths and timescales of demagnetization and magnetization agree well with the measured data.

lifetimes in magnetic materials and, hence, the transport properties of the hot majority and minority spin carriers ${ }^{27-29}$ within the $\mathrm{Ni}$ and Fe layers. Excited minority spin electrons originating in the optical excitation in both the $\mathrm{Al}$ and Ni layers have much shorter lifetimes when passing via superdiffusion through the Ni layer, and are therefore stopped before they can reach the Fe layer. Conversely, majority-spin electrons have much longer lifetimes in $\mathrm{Ni}$ and are able to reach the Fe layer by superdiffusion. Solving the superdiffusion spin-transport equation (see Methods) for electrons excited by a laser pulse of $25 \mathrm{fs}$ and $1.6 \mathrm{eV}$ photon energy in the $\mathrm{Al}, \mathrm{Ni}$ and Fe layers, we predict the transient, layer-specific, normalized magnetizations shown in Fig. 5. The transient response of the Fe layer depends strikingly on the initial relative alignment of the $\mathrm{Ni}$ and Fe layers: for antiparallel magnetization alignment, the stream of spin-majority electrons decreases the net magnetization in the $\mathrm{Fe}$ layer by increasing the amount of spin-minority electrons in Fe. Conversely, for parallel configuration, the superdiffusive spin current of spin majority electrons through the interface increases the number of spin-majority electrons in $\mathrm{Fe}$, thus increasing the net $\mathrm{Fe}$ magnetization and causing opposite polarities in the femtosecond magnetization response of $\mathrm{Ni}$ and Fe layers.

\section{Discussion}

Several competing processes have been proposed for magnetization dynamics on ultrafast timescales ${ }^{5-13}$. For the trilayer systems explored here, we find that the observed magnetization dynamics in the $\mathrm{Ni}$ and buried Fe layer is consistent with superdiffusion of 
excited spin-majority electrons that originate in the $\mathrm{Al}$ and $\mathrm{Ni}$ layers. The experimental data (Fig. 4a,b) agree well with theoretical predictions of superdiffusive spin transport (Fig. 5a,b)-both in terms of the timescales as well as the magnitudes of the demagnetization $(\sim 15 \%$ for $\mathrm{Fe}$ and $\sim 50 \%$ for $\mathrm{Ni})$ and anomalous magnetization increase in $\mathrm{Fe}(\sim 15 \%)$. We note that we have not included spin dissipation channels such as electron-phonon ${ }^{5}$ and electronelectron ${ }^{7}$ spin-flip scattering in our model. This excellent agreement between theory and experiment thus shows that superdiffusion is the dominant process for the observed spin dynamics in the $\mathrm{Ni}$ and Fe layers under the chosen experimental conditions.

The anomalous enhancement of the Fe magnetization is most prominent at relatively low excitation fluence, below $F \approx 2.0 \mathrm{~mJ} \mathrm{~cm}^{-2}$. We find that at higher fluence, direct optical excitation of the Fe layer becomes the dominant source of demagnetization, that is, by increasing the excitation fluence to $F \approx 2.7 \mathrm{~mJ} \mathrm{~cm}^{-2}$, we observe the Fe magnetization to decrease after excitation, even when the $\mathrm{Ni}$ and Fe magnetizations are parallel (see Supplementary Fig. S2). This observation confirms theoretical predictions that the superdiffusive spin current saturates at high excitation powers ${ }^{12,13}$. Conversely, when we reduce the pump fluence to $F \approx 1.3 \mathrm{~mJ} \mathrm{~cm}{ }^{-2}$, we again observe an increase or decrease of magnetization in Fe due to superdiffusive spin transfer, though the effect is weaker than the response shown in Fig. 4.

In summary, by probing ultrafast spin dynamics in interlayer exchange-coupled magnetic structures with elemental specificity using broad bandwidth coherent high harmonic radiation, we are able to elucidate the contribution of spin superdiffusion to the process of ultrafast spin dynamics. Spin superdiffusion leads to a remarkable and counterintuitive magnetization enhancement in $\mathrm{Fe}$ in response to optical pumping when the $\mathrm{Ni}$ and Fe magnetizations are initially parallel. Our discovery provides fundamental insight into spin dynamics on femtosecond timescales, and is relevant for identifying the mechanisms underlying ultrafast spin dynamics. We note that our data demonstrate an ultrafast transfer of angular momentum of longitudinal spin through a spin current on subpicosecond timescales. In contrast, previous works demonstrated spin-transfer torque, that is, a transfer of transverse angular momentum between noncollinear spins and a magnetic moment at microwave frequencies ${ }^{30-33}$, leading to important innovations such as spin-torque magnetic random access memory (RAM) for data storage, spin-torque oscillators for frequency-agile telecommunications, and spin-wave interconnects for spin-based logic. Similarly, we anticipate that superdiffusive spin transfer, which has a considerably larger magnetic moment, can find applications in moving domain walls, switching magnetic nano-elements on subpicosecond timescales or in spin-based electronics operating in the Terahertz frequency range.

\section{Methods}

Sample fabrication and experiment. The $\mathrm{Al}(3 \mathrm{~nm}) / \mathrm{Ni}(5 \mathrm{~nm}) / \mathrm{Ru}(x) / \mathrm{Fe}(4 \mathrm{~nm}) /$ $\mathrm{Ta}(3 \mathrm{~nm})$ multilayers (Al on top) with two different Ru thicknesses $x=1 \mathrm{~nm}$ and $1.5 \mathrm{~nm}$ were fabricated on Si substrates by use of ion beam sputtering. To ensure proper crystal texture, samples were grown on top of a thin Ta layer. Al capping layer prevents oxidation of the magnetic layers. The $\mathrm{Al}$ is self-passivating, and we expect that the top layer actually consists of approximately $1.5 \mathrm{~nm}$ of $\mathrm{AlO}_{x}$ that covers $2 \mathrm{~nm}$ of metallic Al. The entire structure is sufficiently thin for the XUV light to penetrate through and probe the magnetization of the Fe layer. To generate laser high-order harmonics in the XUV, we used a laser amplifier generating laser pulses with $25 \mathrm{fs}$ duration, $1.9 \mathrm{~mJ}$ energy and $1.6 \mathrm{eV}$ photon energy, at $3 \mathrm{kHz}$ repetition rate. Ninety percent of the laser power was focused into a Ne-filled capillary with $150 \mu \mathrm{m}$ diameter to generate HHG spanning $20-72 \mathrm{eV}$ (ref. 34 ), whereas $10 \%$ of the laser energy is used to excite the sample with femtosecond laser pulses with fluencies of, approximately, $2 \mathrm{~mJ} \mathrm{~cm}^{-2}$. The estimated duration of the XUV pulses is $<10 \mathrm{fs}$ (ref. 16), and the XUV light was focused onto the sample using a grazing incidence toroidal mirror. The sample was aligned in the T-MOKE geometry with the magnetization vector of the sample perpendicular to the plane of incidence (see Supplementary Methods). The $p$-polarized XUV probe light is reflected at an angle of $45^{\circ}$ with respect to the sample surface. The XUV photon energy calibration was performed using the Al filter that serves to shield the CCD (charge-coupled device) detector from direct laser light, and has a well-defined absorption edge at $72.6 \mathrm{eV}$.

Theoretical model and calculations. The calculations are based on the superdiffusive spin transport equation ${ }^{12,13}$,

$$
\begin{aligned}
\frac{\partial n(\sigma, E, z, t)}{\partial t}+\frac{n(\sigma, E, z, t)}{\tau(\sigma, E, z)}= & \left(-\frac{\partial}{\partial z} \hat{\phi}+\hat{I}\right) \\
& \times\left(\hat{S} n(\sigma, E, z, t)+S^{\operatorname{ext}}(\sigma, E, z, t)\right)
\end{aligned}
$$

where $n(\sigma, E, z, t)$ is the spin- and energy-dependent density of laser-excited electrons, $\tau(\sigma, E, z)$ is the lifetime, $\hat{\phi}$ and $\hat{I}$ are the electron flux and identity operators, $\hat{S}$ is an integral operator that computes the source term for nextgeneration electrons, which result from elastic, inelastic, as well as cascade processes, and $S^{\text {ext }}(\sigma, E, z, t)$ is the source term containing explicitly the $25 \mathrm{fs}$ pump pulse. The $z$ coordinate is defined as being normal to the layers. The spin- and excitation energy-dependent electronic lifetimes and velocities ${ }^{27,29}$, as well as the ratio of excited majority to minority spin electrons ${ }^{35}$, are taken from $a b$ initio calculations. The femtosecond spin dynamics follows from $m(E, z, t)=2 \mu_{B}[n(\uparrow, E, z, t)-n(\downarrow, E, z, t)]$, with $m(E, z, t)$ the transient spin moment of excited electrons along the depth profile. The material-dependent $M(t)$ is computed as the energy-integrated average of $m(E, z, t)$ over an individual layer.

Partial reflection at the interfaces between two layers has been included. The energy- and spin-dependent reflectivity has been computed assuming the electrons cross the interface as classical particles, with velocity defined by the band structure in each material. The reflected fraction has been obtained by requiring the conservation of linear momentum upon crossing of the interface. All possible multiple reflection paths are taken rigorously into account.

In the calculations of Fig. 5, we assumed a $5 \mathrm{~nm} \mathrm{Ni}$ and $4 \mathrm{~nm}$ Fe layer with a $3 \mathrm{~nm} \mathrm{Al}$ capping layer; however, the Ru layer was not included. The Ru layer, being thin and embedded between the two ferromagnetic layers, will have a spin lifetime and velocity different from those of bulk $\mathrm{Ru}$, therefore, the parameters of this thin $\mathrm{Ru}$ layer are not well known. However, as the Ru layer is very thin, it has only a marginal role in the transport and can to a good approximation be neglected.

\section{References}

1. Beaurepaire, E., Merle, J.- C., Daunois, A. \& Bigot, J.- Y. Ultrafast spin dynamics in ferromagnetic nickel. Phys. Rev. Lett. 76, 4250-4253 (1996).

2. Stamm, C. et al. Femtosecond modification of electron localization and transfer of angular momentum in nickel. Nat. Mater. 6, 740-743 (2007).

3. La-O-Vorakiat, C. et al. Ultrafast soft $\mathrm{x}$-ray magneto-optics at the $M$-edge using a tabletop high-harmonic source. Phys. Rev. Lett. 103, 257402 (2009).

4. Stanciu, C. D. et al. All-optical magnetic recording with circularly polarized light. Phys. Rev. Lett. 99, 047601 (2007).

5. Koopmans, B. et al. Explaining the paradoxical diversity of ultrafast laser induced demagnetization. Nat. Mater. 9, 259-265 (2010).

6. Carva, K., Battiato, M. \& Oppeneer, P. M. Ab initio investigation of the ElliottYafet electron-phonon mechanism in laser-induced ultrafast demagnetization. Phys. Rev. Lett. 107, 207201 (2011).

7. Krauß, M. et al. Ultrafast demagnetization of ferromagnetic transition metals: the role of the Coulomb interaction. Phys. Rev. B 80, 180407 (2009).

8. Mueller, B. Y., Roth, T., Cinchetti, M., Aeschlimann, M. \& Rethfeld, B. Driving force of ultrafast magnetization dynamics. New J. Phys. 13, 123010 (2011).

9. Carpene, E. et al. Dynamics of electron-magnon interaction and ultrafast demagnetization in thin iron films. Phys. Rev. B 78, 174422 (2008).

10. Zhang, G. P., Hübner, W., Lefkidis, G., Bai, Y. \& George, T. F. Paradigm of the time-resolved magneto-optical Kerr effect for femtosecond magnetism. Nat. Phys. 5, 499-502 (2009)

11. Bigot, J.- Y., Vomir, M. \& Beaurepaire, E. Coherent ultrafast magnetism induced by femtosecond laser pulses. Nat. Phys. 5, 515-520 (2009).

12. Battiato, M., Carva, K. \& Oppeneer, P. M. Superdiffusive spin transport as a mechanism of ultrafast demagnetization. Phys. Rev. Lett. 105, 027203 (2010).

13. Battiato, M., Carva, K. \& Oppeneer, P. M. Theory of laser-induced ultrafast superdiffusive spin transport in layered heterostructures. Phys. Rev. B 86, 024404 (2012).

14. Müller, G. M. et al. Spin-polarization in half-metals probed by femtosecond spin excitation. Nat. Mater. 8, 56-61 (2009).

15. Radu, I. et al. Transient ferromagnetic-like state mediating ultrafast reversal of antiferromagnetically coupled spins. Nature 472, 205-208 (2011).

16. Mathias, S. et al. Probing the timescale of the exchange interaction in a ferromagnetic alloy. P. Natl Acad. Sci. USA 109, 4792-4797 (2012).

17. La-O-Vorakiat, C. et al. Ultrafast demagnetization measurements using extreme ultraviolet light: comparison of electronic and magnetic contributions. Phys. Rev. X 2, 011005 (2012).

18. Adam, R., Grychtol, P., Cramm, S. \& Schneider, C. M. Time-resolved measurements of $\mathrm{Ni}_{80} \mathrm{Fe}_{20} / \mathrm{MgO} / \mathrm{Co}$ trilayers in the extreme ultraviolet range. J. Electron Spectrosc. Relat. Phenomena 184, 291-295 (2011). 
19. Ju, G. et al. Ultrafast time resolved photoinduced magnetization rotation in a ferromagnetic/antiferromagnetic exchange coupled system. Phys. Rev. Lett. 82, 3705-3708 (1999).

20. Kaiser, A. M., Wiemann, C., Cramm, S. \& Schneider, C. M. Spatially resolved observation of uniform precession modes in spin-valve systems. J. Appl. Phys. 109, 07D305 (2011).

21. Melnikov, A. et al. Ultrafast transport of laser-excited spin-polarized carriers in $\mathrm{Au} / \mathrm{Fe} / \mathrm{MgO}(001)$. Phys. Rev. Lett. 107, 076601 (2011).

22. Malinowski, G. et al. Control of speed and efficiency of ultrafast demagnetization by direct transfer of spin angular momentum. Nat. Phys. 4, 855-858 (2008).

23. Hecker, M., Oppeneer, P. M., Valencia, S., Mertins, H.-Ch. \& Schneider, C. M. Soft X-ray magnetic reflection spectroscopy at the $3 p$ absorption edges of thin Fe films. J. Electron Spectrosc. Rel. Phenomena 144-147, 881-884 (2005).

24. Grychtol, P. et al. Resonant magnetic reflectivity in the extreme ultraviolet spectral range: interlayer-coupled $\mathrm{Co} / \mathrm{Si} / \mathrm{Ni} / \mathrm{Fe}$ multilayer system. Phys. Rev. $B$ 82, 054433 (2010).

25. Grychtol, P. et al. Layer-selective studies of an anti-ferromagnetically coupled multilayer by resonant magnetic reflectivity in the extreme ultraviolet range. J. Electron Spectrosc. Rel. Phenomena 184, 287-290 (2011).

26. Bigot, J.- V. Spin-sensitive optics. Physics 5, 11 (2012).

27. Zhukov, V. P., Chulkov, E. V. \& Echenique, P. M. GW+T theory of excited electron lifetimes in metals. Phys. Rev. B 72, 155109 (2005).

28. Aeschlimann, M. et al. Ultrafast spin-dependent electron dynamics in fcc Co. Phys. Rev. Lett. 79, 5158-5161 (1997).

29. Zhukov, V. P., Chulkov, E. V. \& Echenique, P. M. Lifetimes and inelastic mean free path of low-energy excited electrons in Fe, Ni, Pt, and $\mathrm{Au}$ : Ab initio GW+T calculations. Phys. Rev. B 73, 125105 (2006).

30. Myers, E. B., Ralph, D. C., Katine, J. A., Louie, R. N. \& Buhrman, R. A. Currentinduced switching of domains in magnetic multilayer devices. Science 285, 867-870 (1999).

31. Tserkovnyak, Y., Brataas, A., Bauer, G. E. W. \& Halperin, B. I. Nonlocal magnetization dynamics in ferromagnetic heterostructures. Rev. Mod. Phys. 77, 1375-1421 (2005)

32. Costache, M. V., Sladkov, M., Watts, S. M., van der Wal, C. H. \& van Wees, B. J. Electrical detection of spin pumping due to the precessing magnetization of a single ferromagnet. Phys. Rev. Lett. 97, 216603 (2006).

33. Woltersdorf, G., Mosendz, O., Heinrich, B. \& Back, C. H. Magnetization dynamics due to pure spin currents in magnetic double layers. Phys. Rev. Lett. 99, 246603 (2007).

34. Kapteyn, H. C., Murnane, M. M. \& Christov, I. P. Extreme nonlinear optics: coherent X-rays from lasers. Phys. Today 58, 39-46 (2005).
35. Oppeneer, P. M. \& Liebsch, A. Ultrafast demagnetization in Ni: theory of magneto-optics for non-equilibrium electron distributions. J. Phys. Condens. Matter 16, 5519-5530 (2004).

\section{Acknowledgements}

The experimental work was funded by the X-Ray Scattering Program of the U.S Department of Energy Office of Basic Energy Science, and made use of facilities provided by the NSF Engineering Research Center in EUV Science and Technology. Financial support by the Swedish Research Council (VR), the European Community's Seventh Framework Programme (FP7/2007-2013) under grant agreement No. 214810, (FANTOMAS), grant agreement No. GA 253316 (International Outgoing Fellowship, S.M.), and the Swedish National Infrastructure for Computing (SNIC) is gratefully acknowledged. We would like to thank Jürgen Lauer, Bernd Küpper, Heinz Pfeifer, Konrad Bickmann and Thomas Jansen for technical support and Bastian Heller for SQUID measurements. We thank Ben Langdon and Neil Anderson at KMLabs Inc. for technical support and Karel Carva and Mirko Cinchetti for discussions.

\section{Author contributions}

D.R., C.L., and M.B. contributed equally to this work, D.R., C.L., E.T. carried out the experiments and data analysis, M.B., P.M., P.M.O. developed the theoretical explanation and performed simulations, R.A., P.G., J.M.S. designed the multilayers and discussed the results, J.M.S. fabricated the samples, C.L., S.M., P.G., R.A., H.C.K., M.M.M. developed the experimental setup and discussed the results, M.B., T.J.S., H.T.N., E.T. calculated the light absorption in the multilayer and discussed the results, M.A., H.C.K., M.M.M., C.M.S., M.B., P.M.O. contributed to the manuscript writing and discussion of results. All co-authors contributed to the data interpretation, internal discussion and the manuscript writing.

\section{Additional information}

Supplementary Information accompanies this paper at http://www.nature.com/ naturecommunications

Competing financial interests: The authors declare no competing financial interests.

Reprints and permission information is available online at http://npg.nature.com/ reprintsandpermissions/

How to cite this article: Rudolf, D. et al. Ultrafast magnetization enhancement in metallic multilayers driven by superdiffusive spin current. Nat. Commun. 3:1037 doi: $10.1038 /$ ncomms2029 (2012). 